ORIGINAL ARTICLE

\title{
Histological features used in the diagnosis of melanoma are frequently found in benign melanocytic naevi
}

\author{
C Urso, F Rongioletti, D Innocenzi, D Batolo, S Chimenti, P L Fanti, R Filotico, R Gianotti, M Lentini, \\ C Tomasini, M Pippione
}

J Clin Pathol 2005;58:409-412. doi: 10.1136/jicp.2004.020933

See end of article for authors' affiliations ...............

Correspondence to: Dr C Urso, Dermatopathology Section, S. M. Annunziata Hospital, I-50011 Antella, Florence, Italy;

cylaur@tin.it

Received for publication 29 June 2004

\begin{abstract}
Aims: The histological features used in the diagnosis of melanoma may be present in benign naevi, but quantitative data are not available. The aim of this study was to establish the real prevalence of such features in naevi.

Methods: Ten dermatopathologists, from nine Italian institutions, studied a series of naevi. Eleven histological parameters currently used in melanoma diagnosis were analysed: asymmetry, poor circumscription, predominance of single melanocytes, irregular confluent nests, suprabasal melanocytes, hair follicle involvement, absence of maturation, cytological atypia, dermal lymphocytic infiltrate, mitoses, and necrosis.

Results: Ninety one naevi were examined: 22 junctional, 59 compound, and 10 intradermal. None of the studied parameters was seen in 22 of the benign naevi studied. One or more investigated features were found in 69 naevi. Poor circumscription was found in 49 cases, single melanocytic predominating in 42 , asymmetry in 41 , irregular confluent nests in 16, cytological atypia in 14, suprabasal melanocytes in seven, and hair follicle involvement in seven; absence of maturation, mitoses and necrosis were not found. Conclusions: The histological features used for the histological diagnosis of melanoma are often present in benign melanocytic naevi. This suggests a critical, non-mechanical use of them in melanoma diagnosis.
\end{abstract}

$\mathrm{T}$ he list of features used to diagnose malignant melanoma includes general architectural features (asymmetry, dimension greater than $6 \mathrm{~mm}$, involvement of epithelial adnexal structures by atypical melanocytes), epidermal features (poor circumscription of the melanocytic proliferation, single melanocytes predominating over nests, suprabasal melanocytes, pleomorphic and confluent melanocytic nests), dermal features (band-like inflammatory infiltrate, lack of maturation of melanocytes with the progressive descent into the dermis), and cytological features (melanocytic atypia, mitotic figures, necrosis of melanocytes). ${ }^{1-5}$ However, although definite data are not available, such features do not appear to be exclusive to melanoma, but may also occur in benign melanocytic naevi. ${ }^{67}$ Our present study analyses a series of benign melanocytic naevi with the aim of establishing the prevalence of some of the histological features currently used in the diagnosis of melanoma.

\section{MATERIALS AND METHODS Dermatopathologist panel}

Our study was undertaken by 10 dermatopathologists, affiliated to the melanocytic lesion group of the Italian Association of Dermatopathology, from nine Italian institutions, namely: Dermatopathology Section, S. M. Annunziata Hospital, Health Unit 10 of Florence (CU); Dermatopathology Centre, Di. S. E. M., University of Genoa (RF); Institute of Dermatology, University "La Sapienza", Rome (DI); Department of Human Pathology, University of Messina (DB, ML); Institute of Dermatology, University "Tor Vergata", Rome (SC); Institute of Dermatology, University of Bologna (PLF); Institute of Dermatology, University of Bari (RF); Institute of Dermatology, University of Milan (RG); and Institute of Dermatology, University of Turin (CT).

\section{Cases studied}

Every participant was invited to retrieve from his/her own files 10 cases (one slide for each case) of melanocytic naevi from adult patients, excluding naevi of palms and soles, and naevi of mammary, genital, and flexural areas. The tissue fragments had been fixed in buffered formalin, routinely processed, and stained with haematoxylin and eosin. The 100 specimens were collected and screened by all the 10 dermatopathologists, in the absence of clinical information. Only cases in which there was a unanimous agreement on the histological diagnosis of benign melanocytic naevus were included in our study. Special naevi referable to a specific diagnostic category (Spitz naevi, Reed naevi, Sutton naevi, recurrent naevi, halo naevi, etc) and technically imperfect slides were excluded. In total, our study analysed 91 naevi. Clinically, lesions had different appearances: some were uniformly brown, with regular and well defined borders; others had colour variegation and/or ill defined borders. Histologically, they had various appearances. Some naevi showed round or oval melanocytes arranged mostly in nests at the dermo-epidermal junction (junctional naevi). ${ }^{8}$ Some showed junctional nests of melanocytes and a prominent lentiginous melanocytic intraepidermal proliferation, with cells arranged as solitary units in elongated rete ridges; melanocytes in the dermis were seen in some lesions (junctional and compound lentiginous naevi). ${ }^{89}$ Some compound and intradermal naevi were exophytic in the shape of a fibroepithelial polyppapilloma (Unna's naevi), or dome shaped, predominantly endophytic (Miescher's naevi) ${ }^{10}$; some compound naevi were slightly raised with nests confined to the dermo-epidermal junction and the papillary dermis; the junctional component tended to extend for several rete ridges beyond the intradermal component of the naevus (Clark's compound naevi). ${ }^{10}$

\section{Definition of histological features}

For each lesion, 11 histological parameters currently used in melanoma diagnosis, for which a consensus by the participants had been achieved in advance, were analysed. 


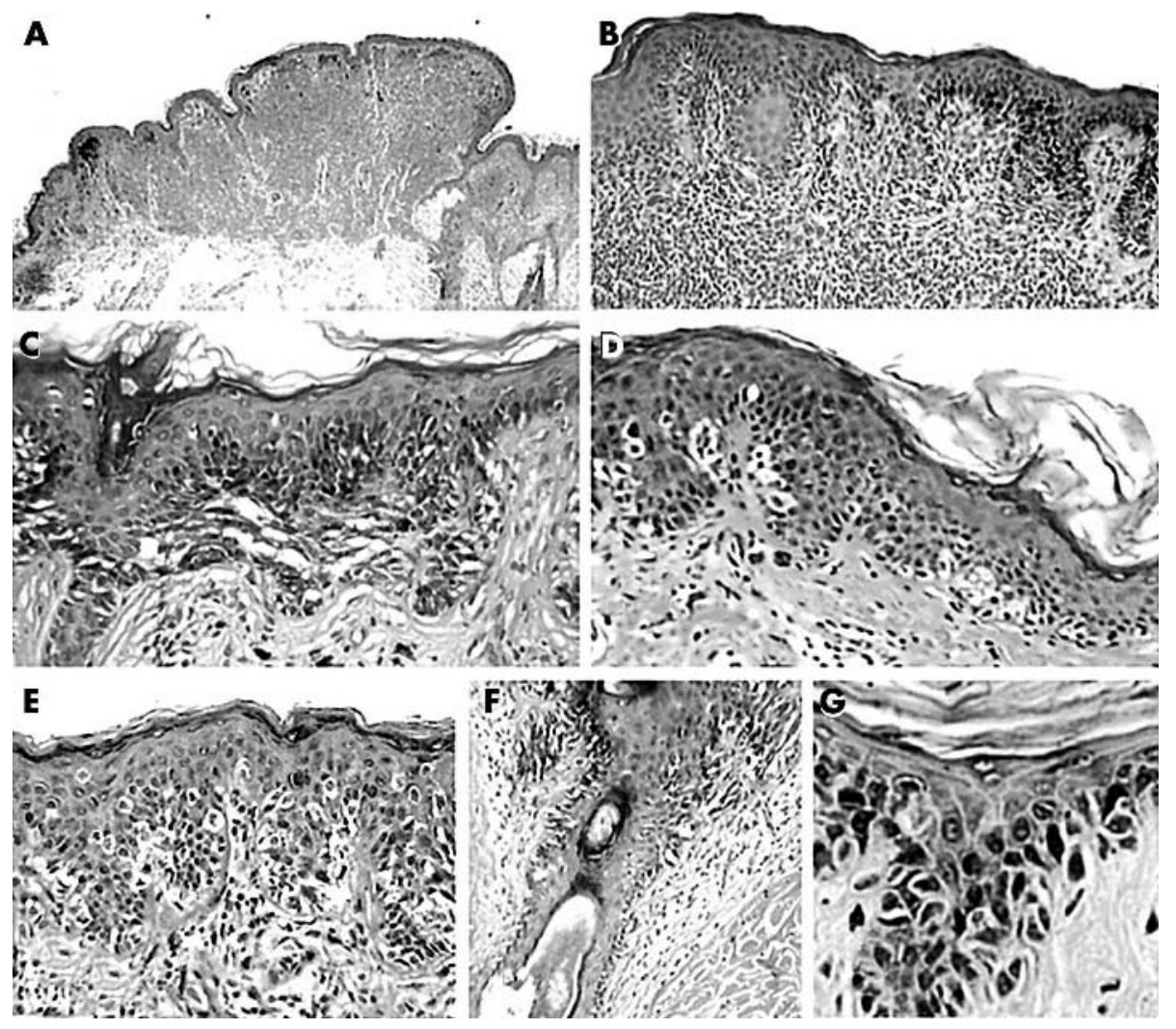

Figure 1 (A) Asymmetry in an intradermal naevus (haematoxylin and eosin (H\&E) stain; original magnification, $\times 25)$. (B) Dermal lymphocytic infiltrate in a lentiginous naevus (H\&E stain; original magnification, $\times 100)$. (C) Confluent nests in a junctional naevus (H\&E stain; original magnification, $\times 200)$. (D) Poor circumscription: the junctional melanocytic proliferation ends with single cells (H\&E stain; original magnification, $\times 200$ ). (E) Predominance of single melanocytes and suprabasal melanocytes (H\&E stain; original magnification, $\times 200)$. (F) Involvement of the hair follicle (H\&E stain; original magnification, $\times 100)$. (G) Cytological atypia in a compound naevus (H\&E stain; original magnification, $\times 300)$.

(1) Asymmetry (fig lA), defined as present when a central line divided the lesion into two parts that looked different in shape, in thickness, or in number and position of dermal cells, ${ }^{3-5}$ absent only when the lesion appeared perfectly symmetrical.

(2) Poor circumscription of the lesion (fig lD), defined as present when the epidermal melanocytic proliferation, extending beyond the dermal component of the lesion, ended with single cells, rather than with a well defined nest. $^{235}$

(3) Predominance of single melanocytes (fig lE), defined as epidermal melanocytes disposed as solitary units predominating over melanocytic nests in some high power fields $\mathrm{s}^{3-5}$; the position of melanocytes in the epidermal layers was not considered.

(4) Irregular and confluent nests (fig 1C), defined as epidermal melanocytic nests, variable in size and in shape and tending to confluence ${ }^{2-5}$; the position of melanocytic nests in the epidermal layers was not considered.

(5) Suprabasal melanocytes (fig 1E), defined as melanocytes above the dermo-epidermal junction $^{3-5}$; the number of suprabasal cells and whether cells appeared single or in nests were not considered.

(6) Involvement of the hair follicle (fig lF), defined as involvement of the hair follicle by melanocytes in the same manner as within the epidermis. ${ }^{5}$

(7) Absence of maturation, defined as failure of the nuclei of melanocytes to become smaller with progressive descent into the dermis..$^{2-5} 8$
(8) Cytological atypia (fig IG), defined as melanocytic nuclei enlarged (more than keratinocytic ones), variable in size and in shape, hyperchromatic, with eosinophilic or amphophilic nucleoli ${ }^{2-5}$; atypia was not graded to avoid subjective evaluations: it was considered as present when it was slight to moderate and when it was severe.

(9) Dermal lymphocytic infiltrate (fig 1B), defined as present when a dermal lymphocytic infiltrate was evident underlying and/or in the context of the lesion ${ }^{8}$; the feature was not graded: it was considered as present when it was sparse and when it was pronounced, forming a continuous band in the dermis.

(10) Mitoses, ${ }^{2-5}$ defined as present when at least one was seen in the dermal component of the lesion.

(11) Necrosis, ${ }^{2-5}$ defined as present when dermal necrotic melanocytes were seen.

\section{Review procedure}

The 9l cases were examined at a multiheaded microscope. Each case was analysed for all the given features, which were evaluated on the basis of a yes/no decision, as present or absent. When a feature could not be evaluated in a given case (for example, absence of maturation in a junctional naevus or in a compound naevus with a superficial dermal component only), it was considered as non-applicable. Each participant individually recorded his/her own evaluation on a special form. None of the investigators knew the other investigators' evaluations. Subsequently, data were collected and 
Table 1 Histological classification of 91 benign melanocytic naevi

\begin{tabular}{lll}
\hline Type of naevus & $\mathbf{N}$ & $\%$ \\
\hline Junctional & 7 & 7.7 \\
Lentiginous, junctional & 15 & 16.5 \\
Lentiginous, compound & 3 & 3.3 \\
Clark's, compound & 24 & 26.4 \\
Miescher's, compound & 29 & 31.8 \\
Miescher's, intradermal & 7 & 7.7 \\
Unna's, compound & 3 & 3.3 \\
Unna's, intradermal & 3 & 3.3 \\
Total & 91 & 100 \\
\hline
\end{tabular}

elaborated. In each lesion, each feature was considered as present if at least six of the 10 observers had agreed on its presence. Non-applicable features were ultimately considered as absent.

\section{RESULTS}

In the series of 91 naevi examined, 22 were junctional, 59 compound, and 10 intradermal. Table 1 gives details of the histological classification. In the 69 compound and intradermal naevi, 53 showed a dermal component, consisting of small rounded dermal naevus cells, arranged in cords and nests, in the papillary dermis, without specific characteristics referable to a special type of naevus, whereas the remaining 16 lesions presented a dermal component showing congenital features (naevus cells disposed in an interstitial or single cell pattern between collagen bundles of at least the upper half of the reticular dermis and in periadnexal, perivascular, and perineural sites). Table 2 gives details of the parameters evaluated in the 91 melanocytic naevi examined.

\section{DISCUSSION}

We found that eight the 11 features investigated were often present in about three quarters of the examined naevi. A quarter of the lesions showed none of the studied features. Asymmetry and poor circumscription of melanocytes, proposed and widely used as diagnostic features of melanoma, ${ }^{34}$ were often seen in naevi. Asymmetry, noted in 41 of the 91 naevi studied here, was previously recorded in $68 \%$ of a series of compound naevi'; poor circumscription of melanocytes, occurring in 49 of our series of junctional, compound and dermal naevi, was noted in $100 \%$ of a series of compound naevi. ${ }^{7}$ Suprabasal melanocytes, one of the most impressive features of melanoma (pagetoid infiltration), especially of the superficial spreading (pagetoid) type, ${ }^{1}$ were detected in seven of the benign melanocytic naevi in our present study and in $7 \%$ of a series of naevi in a previous study. ${ }^{11}$ Suprabasal

Table 2 Prevalence of the parameters evaluated in 91 melanocytic naevi

\begin{tabular}{lll}
\hline Parameters & N & $\%$ \\
\hline Poor circumscription & 49 & 53.8 \\
Predominance of single melanocytes & 42 & 46.1 \\
Asymmetry & 41 & 45.0 \\
Dermal lymphocytic infiltrate & 22 & 24.2 \\
Irregular and confluent nests & 16 & 17.6 \\
Cytological atypia & 14 & 15.4 \\
Involvement of the hair follicle & 7 & 7.7 \\
Suprabasal melanocytes & 7 & 7.7 \\
Absence of maturation & - & 0.0 \\
Mitoses & - & 0.0 \\
Necrosis & - & 0.0 \\
None & 22 & 24.2 \\
\hline
\end{tabular}

melanocytes, improperly considered a synonym of pagetoid infiltration, ${ }^{12}$ are seen in two distinct phenomena occurring in melanocytic lesions: pagetoid infiltration of melanoma ${ }^{3}$ and pseudoinfiltration of naevi. ${ }^{13}$ In our study, hair follicle involvement, frequently noted in melanoma, ${ }^{3}$ especially in the lentigo maligna type, ${ }^{1}$ was detected in seven naevi. Moreover, some of the investigated features commonly used in the diagnosis of melanoma are known to occur in naevi, because they have also been proposed as diagnostic features of the so called dysplastic naevus. ${ }^{14-16}$ Single melanocytic proliferation is quite frequent in benign melanocytic lesions, in which it often presents as a lentiginous pattern, with melanocytes as junctional single units in elongated rete ridges. A lentiginous pattern of melanocytes is seen in lentigo maligna, lentigo maligna melanoma, and acral lentiginous melanoma. ${ }^{1}$ However, lentiginous melanocytic proliferation is seen in several benign lesions as simplex lentigo, lentiginous naevi, acral naevi, and "dysplastic" naevi. ${ }^{14}$ Single melanocytes in a lentiginous pattern, predominating over melanocytic nests, were found in 42 of the 91 naevi analysed in our study, and in $33 \%$ of lesions in another series of benign naevi. ${ }^{11}$ Irregular and confluent nests, included in the list of the diagnostic criteria for melanoma, ${ }^{2}$ have been also considered as a characteristic features of "dysplastic naevi", ${ }^{14-17}$ and were detected in about $17 \%$ of a series including common and "dysplastic" naevi, ${ }^{11}$ a rate very close to that seen in our present study (16 of 91; 17.6\%). Cytological atypia, one of the features noted early in melanoma, ${ }^{18}$ was seen in 14 of the examined naevi, a slightly lower figure that that found in an earlier study (17\%). ${ }^{11}$ However, despite the identical terminology, melanocytic atypia of naevi and melanocytic atypia of melanoma do not appear to be identical. It has been noted that melanomas show fully evolved (severe) atypia, whereas naevi only show slight to moderate atypia, ${ }^{19}{ }^{20}$ although it is not clear whether the differences between slight/moderate atypia and severe (fully evolved) atypia are quantitative or qualitative. It is probable that slight/moderate atypia and severe atypia represent the similar morphological expression of two different processes, rather than the expression of different grades of the same process. ${ }^{21}$ In our study, the features studied were only quantitatively, not qualitatively, evaluated. However, in the series of naevi investigated, the parameters investigated appeared to be less pronounced than is generally seen in melanoma. Asymmetry was slight, cytological atypia slight/moderate, suprabasal melanocytes were low in number and involved the spinous layer only, single melanocytes predominating over nests showed a lentiginous pattern with cells as junctional single units in elongated rete ridges, hair follicles were only slightly involved, and dermal lymphocytic infiltrates were generally sparse.

\section{"In the series of naevi investigated, the parameters investigated appeared to be less pronounced than is generally seen in melanoma"}

It should also be noted that none of the investigated naevi showed absence of maturation, mitoses, or necrosis. Although the series of lesions studied was not large, the results confirm that in skin melanocytic lesions the absence of such features suggests a benign diagnosis; in contrast, absence or impaired maturation and dermal mitoses have been found to be histological features of some variants of melanoma resembling benign naevi (naevoid melanomas). ${ }^{22}$

Data from our study are not meant to detract value from the histological features currently used in the diagnosis of melanoma. However, pathologists should be aware that the histological features they daily search for and use are not 


\section{Take home messages}

- The histological features used for the histological diagnosis of melanoma are often present in benign melanocytic naevi

- Therefore, the diagnostic process should not just involve the mechanical use of a list of histological parameters, but should be a rigorous critical analysis of all the available clinical and histological features of a given lesion

exclusive to melanoma, but in part shared with benign melanocytic naevi. The results of our study provide a quantitative evaluation of the prevalence of such features in benign melanocytic naevi. Data suggest that the diagnostic process cannot just involve the mechanical use of a list of histological parameters, but should be a rigorous critical analysis of all the available clinical and histological features of a given lesion.

\section{Authors' affiliations}

C Urso, Dermatopathology Section, S. M. Annunziata Hospital, Health Unit 10 of Florence, I-50011 Antella, Florence, Italy

F Rongioletti, Dermatopathology Centre, Di. S. E. M., University of Genoa, I-16132 Genoa, Italy

D Innocenzi, Institute of Dermatology, University "La Sapienza", I-00161 Rome, Italy

D Batolo, M Lentini, Department of Human Pathology, University of Messina, I-98125 Messina, Italy

S Chimenti, Institute of Dermatology, University "Tor Vergata", I-00133 Rome, Italy

P L Fanti, Institute of Dermatology, University of Bologna, I-40100 Bologna, Italy

R Filotico, Institute of Dermatology, University of Bari, I-70100 Bari, Italy R Gianotti, Institute of Dermatology, University of Milan, I-20100 Milan, Italy

C Tomasini, M Pippione, Institute of Dermatology, University of Turin, I-10126 Turin, Italy

\section{REFERENCES}

1 McGovern VJ. Melanoma. Histological diagnosis and prognosis. New York: Raven Press, 1983

2 Price NM, Rywlin AM, Ackerman AB. Histologic criteria for the diagnosis of superficial spreading malignant melanoma formulated on the basis of proven metastatic lesions. Cancer 1976; 38:2434-41.

3 Kamino $\mathrm{H}$, Ackerman AB. Malignant melanoma in situ: the evolution of malignant melanoma within the epidermis. In: Ackerman AB, eds. Pathology of malignant melanoma. New York: Masson, 1981:59-91.

4 Roses DF, Harris MN, Ackerman AB. Diagnosis and management of cutaneous malignant melanoma. Philadelphia: WB Saunders, 1983.

5 Dewan $M$, Ackerman AB. What is your diagnosis of these melanocytic neoplasms? Dermatopathology Conceptual and Practical 1999;5:328-35.

6 Okun MR. Silhovette symmetry. An unsupportable histologic criterion for distinguishing Spitz nevi and compound nevi from malignant melanoma. Arch Pathol Lab Med 1997:121:48-53.

7 Okun MR. Histologic demarcation of lateral borders: an unsupportable histologic criterion for distinguishing malignant melanoma from Spitz naevus and compound naevus. Histopathology 1998;33:158-62.

8 Elder DE, Murphy GF. Melanocytic tumors of the skin, Atlas of tumor pathology, 3rd Series, Fascicle 2. Washington, DC: AFIP, 1991.

9 Maize JC, Burgdorf WHC, Hurt MA, et al. Cutaneous pathology. Philadelphia: Churchill Livingstone, 1998.

10 Ackerman AB, Magana-Garcia M. Naming acquired melanocytic nevi Unna's, Miescher's, Spitz's, Clark's. Am J Dermatopathol 1990;12:193-209.

11 Urso C. Atypical histologic features in melanocytic nevi. Am J Dermatopathol 2000;22:391-96

12 Fallowfield ME, Cook MG. Pagetoid infiltration in primary cutaneous melanoma. Histopathology 1992;20:417-20.

13 Urso C. Pseudoinfiltration: an underrecognized feature in melanocytic lesions. Am J Dermatopathol 2002;24:183-4.

14 Elder DE, Greene MH, Guerry D IV, et al. The dysplastic nevus syndrome. Our definition. Am J Dermatopathol 1982;4:455-60.

15 Clark WH Jr, Elder DE, Guerry D IV, et al. A study of tumor progression: the precursor lesions of superficial spreading and nodular melanoma. Hum Pathol 1984;15:1147-65.

16 Dixon SL, Ackerman AB. Some observations, reflections, and questions about dysplastic nevi [editorial]. Am J Dermatopathol (suppl), 1985;7:A17-25.

17 de Wit PEJ, van't Hof-Grootenboer B, Ruiter DJ, et al. Validity of the histopathological criteria used for diagnosis of dysplastic nevi. An interobserver study by the pathology subgroup of the EORTC malignant melanoma cooperative group. Eur J Cancer 1993;29A:831-9.

18 Lever WF, Schaumburg-Lever G. Histopathology of the skin, 6th ed. Philadelphia: JB Lippincott, 1983

19 Rhodes AR, Mihm MC, Weinstock MA. Dysplastic melanocytic nevi: a reproducible histologic definition emphasizing cellular morphology. Mod Pathol 1989;2:306-19.

20 Urso C, Giannini A, Bartolini $M$, et al. Histological analysis of intraepidermal proliferations of atypical melanocytes. Am J Dermatopathol 1990;12:150-5.

21 Urso C. Pagetoid infiltration and pseudoinfiltration-another point of view, Author's reply. Am J Dermatopathol 2002;24:451-52.

22 Zembowicz A, McCusker M, Chiarelli C, et al. Morphologic analysis of nevoid melanoma. A study of 20 cases with a review of the literature. Am J Dermatopathol 2001;23:167-75. 